\title{
Fourier Analysis
}

National Cancer Institute

\section{Source}

National Cancer Institute. Fourier Analysis. NCI Thesaurus. Code C94900.

A mathematical formalism that transforms sequences of values into an equivalent representation that consists of the sum of many sinusoids having different frequencies and amplitudes. 